\title{
Genomic analysis of marginal zone and lymphoplasmacytic lymphomas identified common and disease-specific abnormalities
}

Esteban Braggio ${ }^{1}$, Ahmet Dogan ${ }^{2}$, Jonathan J Keats ${ }^{3}$, Wee J Chng ${ }^{4}$, Gaofeng Huang ${ }^{4}$, Julie M Matthews ${ }^{5}$, Matthew J Maurer ${ }^{2}$, Mark E Law ${ }^{2}$, David S Bosler ${ }^{6}$, Michael Barrett ${ }^{3}$, Izidore S Lossos ${ }^{7}$, Thomas E Witzig ${ }^{2}$ and Rafael Fonseca ${ }^{8}$

${ }^{1}$ Department of Biochemistry and Molecular Biology, Mayo Clinic, Scottsdale, AZ, USA; ${ }^{2}$ Division of Anatomic Pathology, Mayo Clinic, College of Medicine, Rochester, MN, USA; ${ }^{3}$ Translational Genomics, Phoenix, AZ, USA; ${ }^{4}$ Department of Haematology-Oncology, National University Cancer Institute, National University Health System, Singapore, Singapore; ${ }^{5}$ Department of Molecular and Cellular Pharmacology, University of Miami, FL, USA; ${ }^{6}$ Cleveland Clinic, Cleveland, OH, USA; ${ }^{7}$ Division of Hematology-Oncology, Department of Medicine, Sylvester Comprehensive Cancer Center and Department of Molecular and Cellular Pharmacology, University of Miami, FL, USA and ${ }^{8}$ Department of Hematology - Oncology, Mayo Clinic, Scottsdale, AZ, USA

Lymphoplasmacytic lymphomas and marginal zone lymphomas of nodal, extra-nodal and splenic types account for $10 \%$ of non-Hodgkin lymphomas. They are similar at the cell differentiation level, sometimes making difficult to distinguish them from other indolent non-Hodgkin lymphomas. To better characterize their genetic basis, we performed array-based comparative genomic hybridization in 101 marginal zone lymphomas (46 MALT, 35 splenic and 20 nodal marginal zone lymphomas) and 13 lymphoplasmacytic lymphomas. Overall, 90\% exhibited copy-number abnormalities. Lymphoplasmacytic lymphomas demonstrated the most complex karyotype (median = 7 copy-number abnormalities), followed by MALT (4), nodal (3.5) and splenic marginal zone lymphomas (3). A comparative analysis exposed a group of copy-number abnormalities shared by several or all the entities with few disease-specific abnormalities. Gain of chromosomes 3, 12 and 18 and loss of 6q23-q24 (TNFAIP3) were identified in all entities. Losses of 13q14.3 (MIRN15A-MIRN16-1) and 17p13.3-p12 (TP53) were found in lymphoplasmacytic and splenic marginal zone lymphomas; loss of 11q21-q22 (ATM) was found in nodal, splenic marginal zone and lymphoplasmacytic lymphomas and loss of 7q32.1-q33 was found in MALT, splenic and lymphoplasmacytic lymphomas. Abnormalities affecting the nuclear factor kappa $B$ pathway were observed in $\mathbf{7 0} \%$ of MALT and lymphoplasmacytic lymphomas and $30 \%$ of splenic and nodal marginal zone lymphomas, suggesting distinct roles of this pathway in the pathogenesis/progression of these subtypes. Elucidation of the genetic alterations contributing to the pathogenesis of these lymphomas may guide to design-specific therapeutic approaches.

Modern Pathology (2012) 25, 651-660; doi:10.1038/modpathol.2011.213; published online 3 February 2012

Keywords: aCGH; copy-number abnormalities; LPL; MZL; NF-кB pathway

Nodal marginal zone lymphoma, extra-nodal marginal zone lymphoma of MALT, splenic marginal zone lymphoma and lymphoplasmacytic lymphoma

Correspondence: Dr A Dogan, MD, PhD, Division of Anatomic Pathology, Mayo Clinic, College of Medicine, 200 First Street SW, Rochester, MN 55905, USA.

E-mail: ahmet.dogan@mayo.edu

Received 7 September 2011; revised 15 December 2011; accepted 21 December 2011; published online 3 February 2012 are indolent lymphomas accounting for $\sim 10 \%$ of all non-Hodgkin lymphomas. ${ }^{1,2}$ MALT lymphoma is the most frequent subtype, representing $50-70 \%$ of marginal zone lymphomas, followed by splenic and nodal marginal zone lymphomas, which represent $20 \%$ and $10 \%$ of cases, respectively. Lymphoplasmacytic lymphoma, accounting for $1 \%$ of non-Hodgkin lymphomas, is a heterogeneous entity with cytological spectrum ranging from small B-cell lymphocytes, plasmacytoid lymphocytes to mature 
plasma cells and is characterized by frequent involvement of lymph nodes and bone marrow. ${ }^{3}$ MALT lymphomas harbor several characteristic chromosomal abnormalities, including trisomies 3 and 18, deletion of $6 \mathrm{q}$ and a number of translocations, including $\mathrm{t}(11 ; 18)(\mathrm{q} 21 ; \mathrm{q} 21)$ API2-MALT1, $\mathrm{t}(14 ; 18)$ (q32;q21) IGH-MALT1, t(1;14)(p22;q32) IGH-BCL10, $\mathrm{t}(3 ; 14)(\mathrm{q} 27 ; \mathrm{q} 32)$ and $\mathrm{t}(3 ; 14)(\mathrm{p} 14.1 ; \mathrm{q} 32)$ IGH-FOXP1. ${ }^{4-12}$ The translocations are mutually exclusive, but often they are present in conjunction with other genomic abnormalities, with the exception of the $\mathrm{t}(11 ; 18)$ API2MALT1, which usually is the sole chromosomal abnormality. ${ }^{13}$ Interestingly, at least four of these translocations cause overexpression of MALT1 or $B C L 10$, leading to constitutive activation of the nuclear factor kappa B (NF- $\mathrm{B})$ signaling pathways-a common pathogenesis pathway in MALT lymphomas. ${ }^{14-16}$ However, depending on the anatomic location, $25-75 \%$ of MALT lymphomas do not harbor any of these chromosomal translocations.

Knowledge surrounding the genetic events characterizing splenic, nodal and lymphoplasmacytic lymphoma is more limited. The splenic marginal zone lymphoma is mainly characterized by the presence of recurrent deletions on $7 q 32-q 35 .{ }^{11,17-19}$ Whole or partial gains of chromosomes 3 and 12 and losses of $17 p$ have also been identified in this entity. ${ }^{18,20}$ In the nodal marginal zone lymphoma some initial studies have highlighted the presence of whole or partial gains on chromosomes $3,6 \mathrm{p}, 12$ and $18,{ }^{10}$ but no unique abnormalities have been identified. Finally, few recurrent abnormalities (6q deletion, $6 \mathrm{p}$ gain and whole or partial gains on chromosomes 3,4 and 18) have been identified in lymphoplasmacytic lymphoma, mainly in cases associated with Waldenström's macroglobulinemia. ${ }^{21-23}$

Consequently, there is a limited knowledge regarding the pathogenesis of these diseases and what are the genetic events contributing to malignant transformation. The aim of this study was to perform a comprehensive high-resolution genomic analysis in a large series of marginal zone/lymphoplasmacytic lymphoma to better characterize the genetic basis of the different entities and identify molecular mechanisms involved in the pathogenesis of these indolent B-cell non-Hodgkin lymphomas.

\section{Materials and methods}

\section{Patient Samples}

In all, 114 patients were included in this study: 46 MALT lymphomas (22 pulmonary, 11 salivary glands, 7 lacrimal glands and 6 gastrointestinal), 35 splenic marginal zone lymphomas, 20 nodal marginal zone lymphomas and 13 non-Waldenström's macroglobulinemia lymphoplasmacytic lymphomas. All cases were reviewed before the study on paraffin sections with immunohistochemistry. Sections of each frozen tissue used for the study were also reviewed by histological examination and immunohistochemistry. All cases were diagnosed according to the criteria established by the 2008 World Health Organization Classification of Lymphoid Neoplasms. ${ }^{24}$

The Waldenström's macroglobulinemia cases were excluded from this study. The cases fulfilling the criteria for Waldenström's macroglobulinemia according to the 2008 World Health Organization Classification and International Workshop On Waldenström's Macroglobulinemia ${ }^{25,26}$ were published in a previous study. ${ }^{21}$ The diagnostic criteria for Waldenström's macroglobulinemia are the presence of (i) a lymphoplasmacytic lymphoma morphology as described in the 2008 World Health Organization classification, (ii) bone marrow involvement and (iii) any level of IgM paraprotein. The cases included in this study either lacked evidence of bone marrow involvement or IgM paraprotein. Of the 13 cases of lymphoplasmacytic lymphomas analyzed in this study, 6 cases lacked a monoclonal gammopathy at presentation. Remaining cases either had IgG or IgA gammopathy or IgM gammopathy without evidence of the bone marrow involvement. The lymphoplasmacytic lymphomas cases were composed of a mixture of small lymphocytes, small lymphoplasmacytoid cells and occasional plasma cells. Architecturally the infiltrate was diffused with a predominantly paracortical and medullary pattern often sparing the medullary sinuses. In contrast, the nodal marginal zone lymphoma cases contained intermediate sized cells with abundant pale cytoplasm (so-called monocytoid cytology). They lacked lymphoplasmacytoid cells and plasma cell differentiation was minimal or absent. The cases that cannot be clearly classified into these two categories were not included in the study. Finally, the so-called variants such as splenic lymphoma villous lymphocytes or splenic B-cell lymphoma unclassified were not studied.

In all, 8 of the 46 MALT lymphomas were previously shown to have t(11;18)(q21;q21) API2-MALT1 by FISH analysis. ${ }^{27}$ Tumor purity was estimated by percent of CD20-positive cells in the tumors with a median of $70 \%$ (range $20-100 \%$ ). Tissues analyzed of each case are shown in Supplementary Table S1. Biospecimens were collected after informed consent was obtained in accordance with the Declaration of Helsinki in each of the participating centers and the study was conducted following the Institutional Review Board approval in all the centers.

\section{Array-Based Comparative Genomic Hybridization}

Genomic DNA was obtained from snap-frozen tissue using standard phenol-chloroform extraction methods. High-resolution array-based comparative genomic hybridization was performed with the Human Genome 
244A microarray (Agilent Technologies, Palo Alto, CA, USA). The digestion, labeling and hybridization steps were done as previously described with minor modifications. ${ }^{21}$ Briefly, $1.2 \mu \mathrm{g}$ of tumor and reference DNAs were separately digested with Bovine DNaseI (Ambion, Austin, TX, USA) for $12 \mathrm{~min}$ at room temperature. Random primers and exoKlenow fragment (Invitrogen, Carlsbad, CA, USA) were used to differentially label tumor (Cy5) and reference (Cy3) genomic DNA samples (GE Healthcare, Piscataway, NJ, USA). Labeled genomic reactions were cleaned-up by purification columns (Invitrogen) and hybridized at $65^{\circ} \mathrm{C}$ for $40 \mathrm{~h}$. Microarrays were scanned in a DNA Microarray Scanner (Agilent Technologies). Feature extraction was performed with Feature extraction Software, version 9.5 (Agilent Technologies). Log2 ratio data were imported and analyzed using DNA Analytics Software version 4.0.85 (Agilent Technologies).

Copy-number abnormalities were calculated using aberration detection module 1 algorithm ${ }^{28}$ with a threshold of 7.5. A 2 probe, 0.25 log2 filters were used in the aberration detection, obtaining an average genomic resolution of $17 \mathrm{~Kb}$. Copy-number variations were identified and excluded from the analysis as previously described. ${ }^{21}$

Unsupervised hierarchical clustering and Pearson's correlation were performed using Genespring software. An in house algorithm was used to represent the penetrance plots.

\section{FISH}

FISH DNA probes to validate copy-number changes affecting TNFAIP3 was selected for a fosmid clone using the UCSC genome browser. The normal cutoff for scoring FISH probes was determined using normal controls and was of $10 \%$. In all, 100 cells from formalin-fixed paraffin embedded tissues were scored in each case. The specificity of each probe at chromosome and gene level was confirmed by hybridization to normal metaphase preparations and by gene-specific PCR, respectively. A list of probes used and chromosomal localization is provided in Supplementary Table S2.

\section{DNA Sequencing}

Genome sequencing was performed on the TNFAIP3 coding exons and adjacent intron-exon junctions in all lymphoplasmacytic lymphomas and in 20 MALT lymphomas patients. All the coding regions were amplified using $10 \mathrm{ng}$ of genomic DNA in $25 \mu \mathrm{l}$ reactions. The specific primers used in this study are listed in Supplementary Table S3. Capillary electrophoresis was performed on an ABI3730 sequencer (Applied Biosystems, Foster City, CA, USA). DNA sequences were analyzed using Sequencher V4.5.

\section{Statistic Analysis}

Genomic complexity, defined as the total size across all detected gains, and likewise, across all detected losses, was evaluated both as a continuous variable and as a categorical variable. ANOVA and two-tailed $t$-test were used to test for associations. Statistical significance was considered when $P<0.05$.

\section{Results}

\section{Overview of Copy-Number Abnormalities}

We performed array-based comparative genomic hybridization in 13 non-Waldenström's macroglobulinemia lymphoplasmacytic lymphomas and 101 marginal zone lymphoma patients, including 46 MALT lymphomas, 35 splenic and 20 nodal marginal zone lymphomas. A total of 90\% (103 of 114) cases had copy-number abnormalities, ranging from $85 \%$ of nodal marginal zone lymphomas to $92 \%$ of lymphoplasmacytic lymphomas. The number of copy-number abnormalities was used as a measurement of the genomic complexity of each patient sample (Supplementary Table S4). Overall, 719 copy-number abnormalities were found, comprising 427 losses and 292 gains. Homozygous deletions and multiple copy gains were rare (17 biallelic deletions and 23 2-3 extra copy gains, respectively). Conversely, no gene amplifications ( $>3$ extra copies) were found. Lymphoplasmacytic lymphomas was the entity with the largest number of abnormalities (median of 7 copy-number abnormalities per sample; range: 0-44), followed by MALT lymphomas without $\mathrm{t}(11 ; 18)(\mathrm{q} 21 ; \mathrm{q} 21)(4 ; 0-18)$, nodal $(3.5 ; 0-$ 35), splenic (3; 0-66) and finally MALT lymphomas with $\mathrm{t}(11 ; 18)(\mathrm{q} 21 ; 21)(1 ; 0-4)$ (Table 1). A graphic representation of all abnormalities detected per entity, type (gains and losses), prevalence and chromosome location is shown in Figure 1. The complete set of abnormalities is described in Supplementary Table S5.

It is remarkable that, even using this highresolution microarray platform, MALT lymphomas with the $\mathrm{t}(11 ; 18)(\mathrm{q} 21 ; \mathrm{q} 21)$ show a very stable karyotype, without any recurrent abnormality with the exception of losses flanking MALT1 and BIRC3, which are a cytogenetic consequence of unbalanced t(11;18)(q21;21) translocations (Figure 2 and Supplementary Tables S4 - S5). For this reason, unless specified, MALT lymphoma patients with $t(11 ; 18)-$ (q21;q21) were excluded from the remaining analyses of this study.

\section{Common Abnormalities to All Entities}

In all, 8 abnormalities were recurrently found in all entities: whole or partial gains of chromosomes 3, 12 and 18, gain of 1q23-q25 and deletions of cytobands 1p21-p22, 1q42, 6q23.3-q24.1 and 13q14 (Figure 1 
Table 1 Summary of number and size of abnormalities per entity

\begin{tabular}{|c|c|c|c|c|}
\hline Entity & $\mathrm{N}$ & $\begin{array}{c}\text { Median number of copy-number } \\
\text { abnormalities per case }\end{array}$ & $\begin{array}{l}\text { Mean number of copy-number } \\
\text { abnormalities per case }\end{array}$ & Range \\
\hline $\begin{array}{l}\text { Non-Waldenstrom's macroglobulinemia } \\
\text { lymphoplasmacytic lymphoma }\end{array}$ & 13 & 7 & 11 & $3-21$ \\
\hline Nodal marginal zone lymphomas & 20 & 3.5 & 6 & $0-35$ \\
\hline Splenic marginal zone lymphomas & 35 & 3 & 7 & $0-66$ \\
\hline MALT lymphomas & 46 & 3 & 4 & $0-18$ \\
\hline MALT without $\mathrm{t}(11 ; 18)$ & 38 & 4 & 5 & $0-18$ \\
\hline MALT with t(11;18) & 8 & 1 & 2 & $0-4$ \\
\hline
\end{tabular}
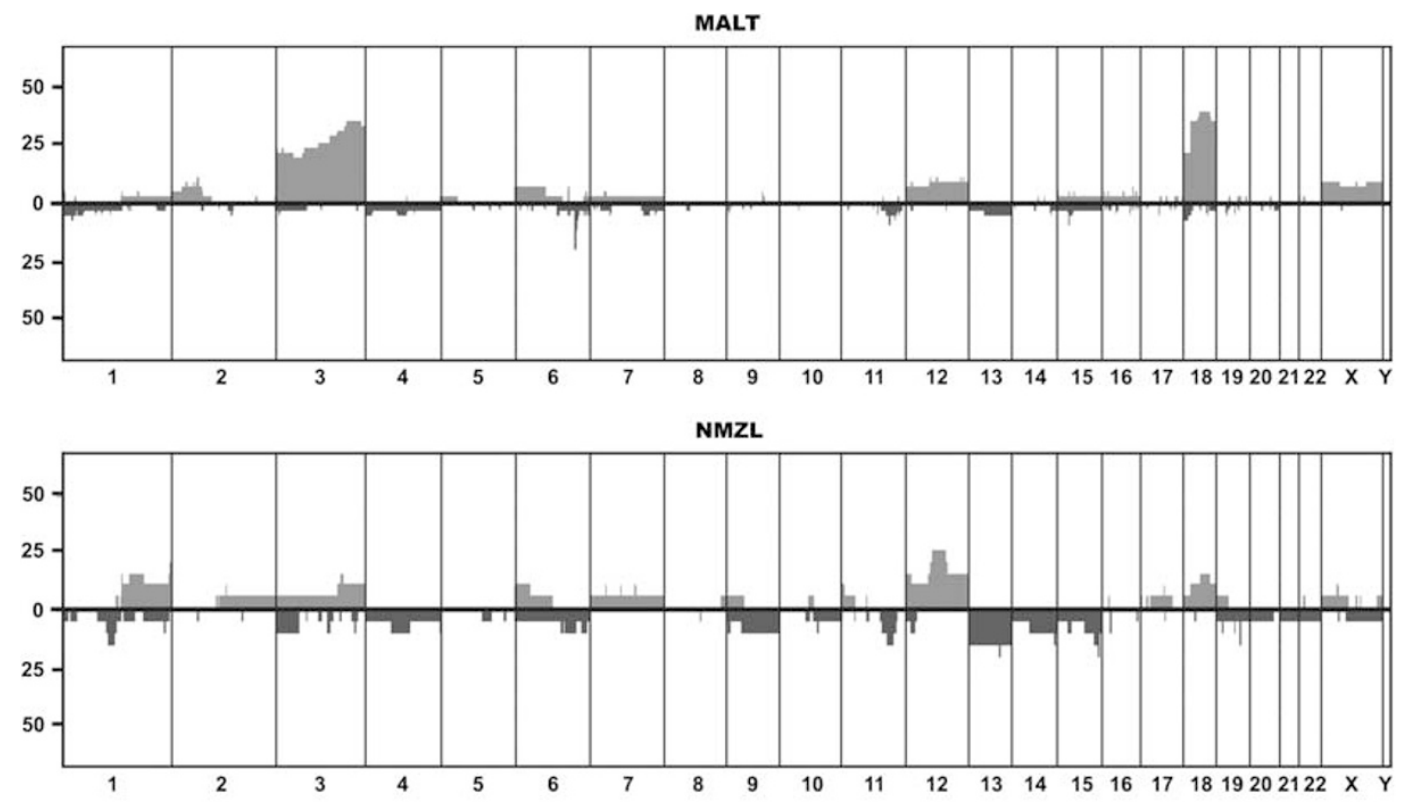

SMZL

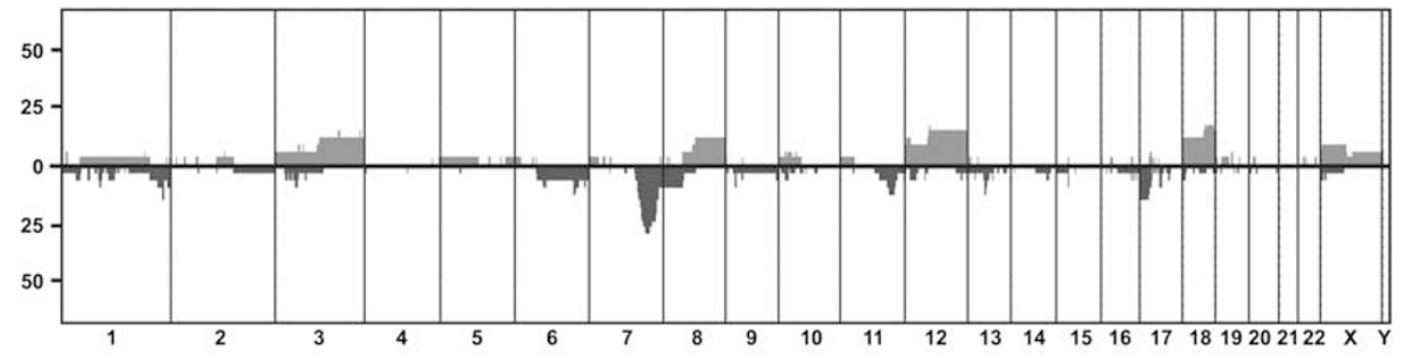

LPL

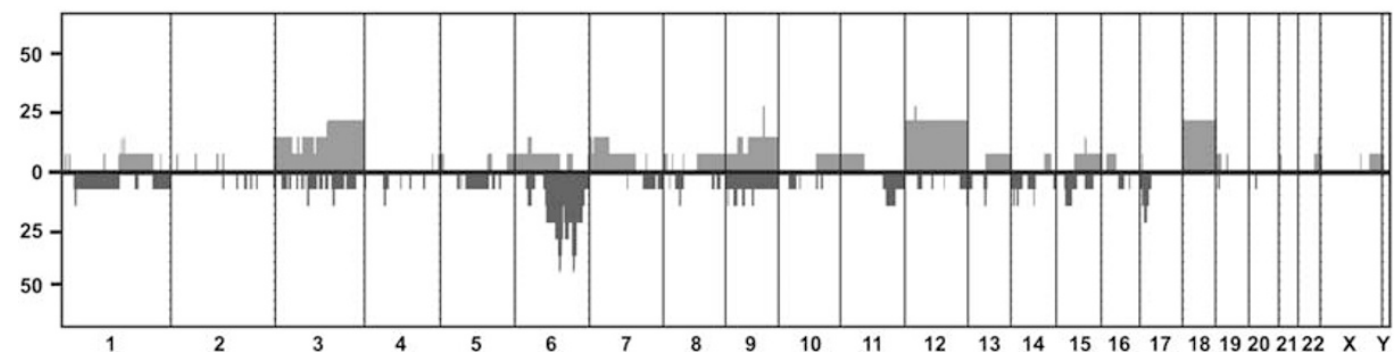

Figure 1 Overview of the copy-number abnormalities identified in marginal zone and lymphoplasmacytic lymphomas. Chromosomes 1 to Y are represented from left to right. Light gray blocks represent chromosome gains, whereas dark gray blocks represent chromosome losses. The amplitude in each abnormal region represents the frequency (\%) of each copy-number abnormality. 

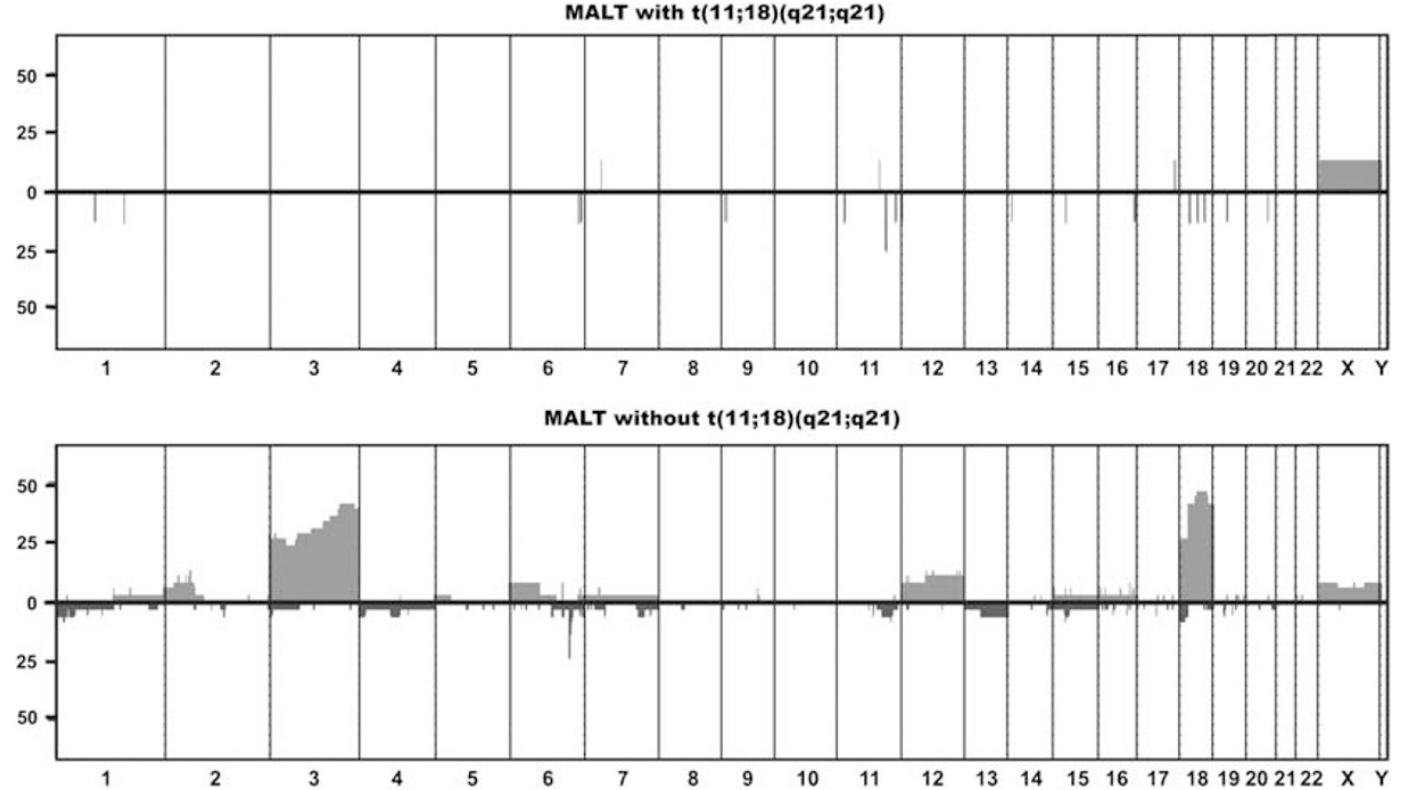

Figure 2 Copy-number abnormality comparison between MALT lymphomas with and without t(11;18) API2-MALT1. Chromosomes 1 to $\mathrm{Y}$ are represented from left to right. Light gray blocks represent chromosome gains, whereas dark gray blocks represent chromosome losses. The amplitude in each abnormal region represents the frequency (\%) of each copy-number abnormality.

Table 2 List of minimal deleted and minimal gained regions identified in $>10 \%$ of cases in one or more entities

\begin{tabular}{|c|c|c|c|c|c|c|c|}
\hline Event & Cytoband & Start (bp) & Stop (bp) & Size $(M b)$ & $\begin{array}{l}\text { Nodal Lymphoplasmacytic } \\
\text { marginal zone lymphomas (13) } \\
\text { lymphomas } \\
(20)\end{array}$ & $\begin{array}{l}\text { Splenic } \\
\text { marginal } \\
\text { zone } \\
\text { lymphomas } \\
(35)\end{array}$ & $\begin{array}{l}\text { MALT } \\
\text { lymphomas } \\
(38)\end{array}$ \\
\hline
\end{tabular}

\begin{tabular}{|c|c|c|c|c|c|c|c|c|}
\hline CN loss & 1p21.2-p22.1 & 94131585 & 103829936 & 9.7 & 15 & 7.7 & 5.7 & 2.6 \\
\hline CN loss & $1 \mathrm{q} 42.3$ & 232809474 & 233657080 & 0.85 & 10 & 7.7 & 14.3 & 2.6 \\
\hline CN loss & $6 q 21$ & 105423847 & 110194804 & 4.77 & 10 & 46.1 & 5.7 & 2.6 \\
\hline CN loss & $6 q 22.31$ & 119694067 & 120249386 & 0.56 & 10 & 38.5 & 5.7 & 2.6 \\
\hline CN loss & $6 \mathrm{q} 23.3-\mathrm{q} 24.1$ & 137982509 & 138292603 & 0.31 & 10 & 46.1 & 11.4 & 21.1 \\
\hline CN loss & $6 \mathrm{q} 24.2$ & 143040435 & 143616681 & 0.58 & 5 & 30.8 & 8.6 & 10.5 \\
\hline CN loss & $7 \mathrm{q} 32.1-\mathrm{q} 33$ & 127287795 & 133367246 & 6.08 & 0 & 7.7 & 26 & 5.2 \\
\hline CN loss & $11 q 21-q 22$ & 97092852 & 109858814 & 12.77 & 15 & 15.4 & 11.4 & 0 \\
\hline CN loss & $13 q 14.3$ & 49485198 & 49917506 & 0.43 & 15 & 15.4 & 11.4 & 2.6 \\
\hline CN loss & 14q12-q13.1 & 30878740 & 32266770 & 1.39 & 0 & 15.4 & 0 & 0 \\
\hline CN loss & $15 \mathrm{q} 25.3-\mathrm{q} 26.2$ & 85029553 & 93129421 & 8.1 & 15 & 0 & 0 & 2.6 \\
\hline CN loss & 17p13.1 & 7149131 & 8106985 & 0.96 & 0 & 23.1 & 14.3 & 0 \\
\hline $\mathrm{CN}$ gain & 1q23.3-q25.3 & 157659528 & 184755949 & 27.1 & 15 & 7.7 & 2.8 & 0 \\
\hline CN gain & 2p15-p16 & 58802364 & 61259418 & 2.46 & 0 & 7.7 & 2.8 & 10.5 \\
\hline $\mathrm{CN}$ gain & $3 q 23-q 24$ & 143871135 & 148165451 & 4.29 & 15 & 23.1 & 11.4 & 36.9 \\
\hline CN gain & 6p22.1-p25.3 & 1 & 29185959 & 29.19 & 10 & 7.7 & 0 & 10.5 \\
\hline CN gain & $8 q 23.3-q 24.22$ & 114625817 & 136133311 & 19.19 & 0 & 7.7 & 11.4 & 0 \\
\hline $\mathrm{CN}$ gain & $9 q 21.32-q 34.3$ & 85708336 & 140241876 & 54.53 & 0 & 15.4 & 0 & 0 \\
\hline CN gain & $12 q 13.13-q 21.31$ & 51647698 & 80538769 & 28.89 & 25 & 23.1 & 14.3 & 5.2 \\
\hline $\mathrm{CN}$ gain & $18 \mathrm{q} 21.2-\mathrm{q} 21.33$ & 49712041 & 58970878 & 9.26 & 15 & 23.1 & 17.1 & 39.5 \\
\hline
\end{tabular}

Copy-number abnormalities present in $10-20 \%$ are in light gray boxes and copy-number abnormalities $>20 \%$ are in dark gray boxes.

and Table 2). Unsupervised hierarchical analysis shows clusters based on the main abnormalities but without obtaining a good differentiation between lymphoma entities (Supplementary Figure S1). Whole or partial gains of chromosomes 3 and 18 were the most common abnormalities seen in MALT lymphomas (37\% and $40 \%$, respectively) and one of the most frequent abnormalities in the remaining diseases, ranging from 15 (chromosome 3) and 23\% (chromosome 18) in lymphoplasmacytic lymphomas to $15 \%$ of each in nodal marginal zone lymphomas (Table 2). The minimal gained regions were located at 3q23-q24 (4.3 Mb) and 18q21.2-q21.33 (9.3 Mb), the last one including MALT1 but not BCL2. 
The concomitant gain of chromosomes 3 and 18 was observed in MALT lymphomas $(P=0.002)$ and lymphoplasmacytic lymphoma $(P=0.002)$ but not in nodal or splenic marginal zone lymphomas $(P>0.05)$.

The prevalence of whole or partial gain of chromosome 12 ranges from 5\% in MALT lymphomas to $25 \%$ in nodal marginal zone lymphomas and a minimal gain region of $29 \mathrm{Mb}$ was refined on 12q13.13-q21.31 (Table 2). Deletion of 6q was the most common abnormality in lymphoplasmacytic lymphoma (46\%). This deletion was also present in a substantial number of MALT lymphomas $(21 \%)$ and less frequently in splenic and nodal marginal zone lymphomas (Figure 1 and Table 2). Lymphoplasmacytic lymphoma was primarily characterized by loss of the entire $6 \mathrm{q}$ arm. In a subset of lymphoplasmacytic lymphomas, focal deletions were identified and four minimal deleted regions were defined on 6q21, 6q22.31, 6q23.3-q24.1 and 6q24.2, the third of them overlapping with the minimal deleted region identified in MALT lymphomas. Biallelic and focal monoallelic deletions on 6q23.3-q24.1 identified TNFAIP3 as the sole gene inside the common minimal deleted region in all the entities (Supplementary Figure 2a), subsequently confirmed by interphase FISH (Supplementary Figure 2b). TNFAIP3 biallelic deletions were identified in lymphoplasmacytic lymphomas and all marginal zone lymphoma entities except splenic marginal zone lymphomas (Supplementary Figure 2c). In MALT lymphomas, biallelic deletions were identified in lacrimal glands/ocular adnexa (2 of 6 cases) and salivary glands (2 of 12), and focal monoallelic deletions were found in salivary glands (1 of 12), stomach (1 of 2), bowel (1 of 3 ) and lung (1 of 15)(Supplementary Figure 2d). All the coding regions of TNFAIP3 were sequenced in 20 MALT and 13 lymphoplasmacytic lymphoma cases, but no mutations were identified.

Chromosome 13 deletions were observed in all entities, but the affected region varies between entities. Nodal marginal zone and MALT lymphomas were characterized by whole chromosome 13 deletions, as is observed in myeloma, whereas focal deletions on 13q14.3, including the microRNAs MIR15A and MIR16-1, were identified in lymphoplasmacytic and splenic marginal zone lymphomas (Figure 1). This focal region is equivalent to the most common copynumber abnormality in chronic lymphocytic leukemia.

\section{Recurrent Abnormalities in Lymphoma Subsets}

Several chromosomal regions were involved in recurrent imbalances in more than one lymphoma entity but were absent in others (Figure 3). Seventeen minimal deleted and nine minimal gain regions were defined based on the presence in at least one entity with a frequency of $\geq 10 \%$ (Table 2 ).

Deletion of 7q32.1-q33 was the most common abnormality in splenic marginal zone lymphoma

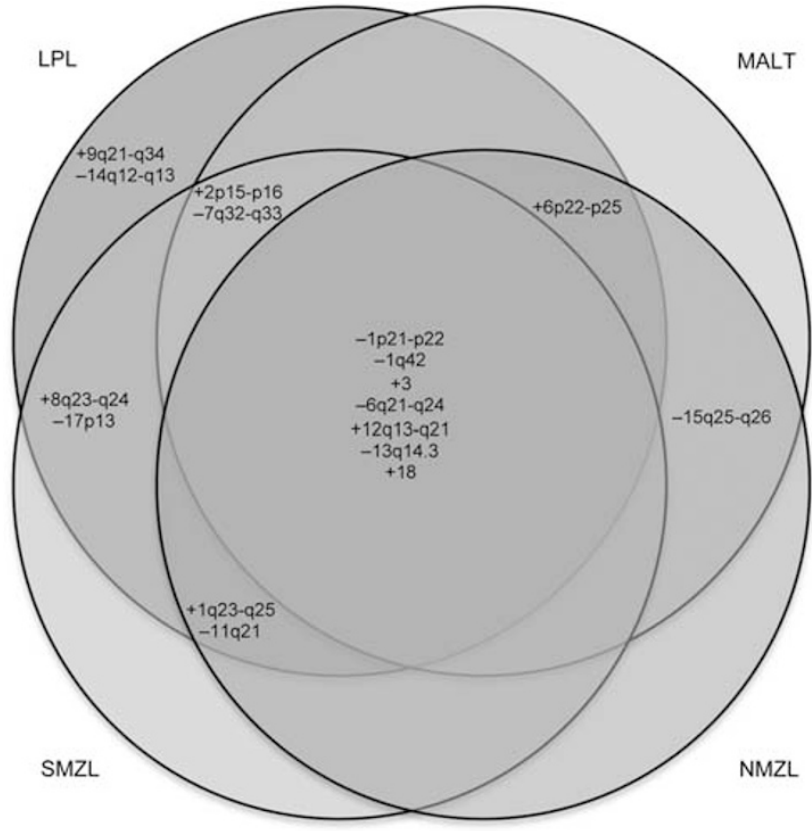

Figure 3 Venn diagram summarizing the common and distinct copy-number abnormalities between entities. Abnormalities found in $>10 \%$ of at least one entity are shown.

( $26 \%)$, but it was also identified in lymphoplasmacytic lymphomas (8\%) and MALT lymphomas (5\%), but not in nodal marginal zone lymphomas (Figure 1 and Table 2). A $14.8 \mathrm{Mb}$ minimal deleted region including 81 genes and 10 microRNAs was delineated (Supplementary Table S6). In all cases, the abnormality affected only one allele. The number of molecular alterations in splenic marginal zone lymphoma suggests a significant degree of molecular heterogeneity. Splenic marginal zone lymphomas with deletion of 7q32.1-q33 were characterized for having a slight, but not statistically significant, increase in the number of copy-number abnormalities per karyotype (median of 6 copy-number abnormalities per karyotype) when compared to patients without deletion of $7 \mathrm{q}$ (median of 3 abnormalities). Conversely, several recurrent abnormalities were only identified in the group without deletion of $7 \mathrm{q}$ such as deletion of $13 \mathrm{q} 14.3$ (16\% of patients without deletion), whole or partial gain of chromosome 18 (24\%) and partial gains of Xp (12\%) (Supplementary Figure S3).

Deletion of $17 \mathrm{p} 13.3-\mathrm{p} 12$, containing TP53, was identified in lymphoplasmacytic (15\%) and splenic marginal zone lymphomas (14\%), but not in MALT or nodal marginal zone lymphomas. This abnormality was associated with a significantly more complex karyotype in splenic marginal zone lymphomas cases (median of 25 versus 3 copy-number abnormalities in cases without $17 p$ deletion; $P<0.0001$ ). The presence of deletion of $7 q$ was not correlated with deletion of $17 p$ in splenic marginal zone lymphoma cases. 
Recurrent deletions on 11q21-q22 were identified in all lymphoma entities, with the exception of MALT lymphomas, ranging from $15 \%$ in nodal to $11 \%$ in splenic marginal zone lymphomas (Table 2). A $12.77 \mathrm{Mb}$ minimal deleted region was delineated, including $A T M$ and 45 additional genes.

Focal deletions on 9p21.3 including CDKN2A were identified in lymphoplasmacytic, splenic and nodal marginal zone lymphoma patients, but not in MALT lymphomas. Recurrent gains on 1q23.3-q25.3 were identified in lymphoplasmacytic and nodal marginal zone lymphoma. Deletion of 1p21.2-p22.1 was mainly observed in nodal marginal zone lymphoma (15\%), but also in low frequency of splenic and lymphoplasmacytic lymphoma cases. Whole or partial gains on $6 \mathrm{p}$ were common to all entities with the exception of splenic marginal zone lymphoma.

Finally, some abnormalities were only recurrent in one lymphoma entity. Gain of chromosome 9 was only found in lymphoplasmacytic lymphomas. Deletion of 15q25.3-q26.2 was the second most common abnormality found in nodal marginal zone lymphoma $(15 \%)$, but was absent in the remaining entities, with the exception of one MALT lymphoma case. (Figure 1 and Table 2).

\section{Copy-Number Abnormalities Affecting Regulators of the NF-kB Pathway}

In addition to $17 \%$ of cases with $\mathrm{t}(11 ; 18)$, extra copies of MALT1 (18q21) and/or focal gains of REL (2p15-p16) and/or monoallelic/biallelic deletions affecting TNFAIP3 (6q23) were identified in an additional $46 \%$ of MALT lymphomas, totaling $63 \%$ of MALT lymphoma cases with abnormalities affecting regulatory genes from the NF- $\kappa \mathrm{B}$ signaling pathway (Figure 4a). Depending on the anatomic site, the prevalence of NF- $\mathrm{BB}$ abnormalities varies from $50 \%$ in lacrimal glands to $75 \%$ in salivary glands (Figure 4b). These abnormalities were identified alone or in combination, but all of them were mutually exclusive with the $t(11 ; 18)(q 21 ; 21)$.

In lymphoplasmacytic lymphomas, no translocations leading to the activation of the NF- $\mathrm{kB}$ signaling pathway have been described. However, deletions of
TNFAIP3, TRAF3 and gains of MALT1 and REL alone or in combination were identified in $61.5 \%$ of cases (8 of 13 patients) (Figure 4a). Abnormalities affecting the major regulatory genes of the $\mathrm{NF}-\kappa \mathrm{B}$ signaling pathways were identified in a significantly lower frequency in nodal $(30 \%)$ and splenic marginal zone lymphomas (31\%).

\section{Discussion}

In this study we performed a comprehensive, highresolution, copy-number analysis of non-Hodgkin lymphomas including MALT, nodal and splenic marginal zone lymphomas and the related entity lymphoplasmacytic lymphoma. The analysis of the genomic complexity based on the number and size of copy-number abnormalities shows a range from a very stable karyotype in MALT lymphomas harboring the $\mathrm{t}(11 ; 18)$ API2-MALT1 to more complex karyotypes such as those seen in lymphoplasmacytic lymphoma. However, overall this subset of lymphomas demonstrates low complexity compared with that observed in more aggressive B-cell diseases. ${ }^{29-32}$ The low karyotypic complexity observed in MALT lymphoma cases with the $\mathrm{t}(11 ; 18)$ API2-MALT1 is remarkable. Previous studies using lower resolution approaches have proposed that the classical translocations in MALT lymphomas are present in combination with other genetic abnormalities, with the exception of the $t(11 ; 18)$ API2-MALT1, which has been the sole abnormality ${ }^{13}$ associated with a very stable karyotype. ${ }^{33}$ In this study we identified a median of one extra abnormality per karyotype harboring the $\mathrm{t}(11 ; 18)$ API2-MALT1, but we did not identify any recurrent abnormality other than focal copy-number abnormalities surrounding API2 and MALT1, which are a consequence of unbalanced translocations. Thus, our data confirm the presence of an extremely conserved karyotype in MALT lymphomas with $\mathrm{t}(11 ; 18)$ API2-MALT1, even given an analysis of the genome with an average resolution of $17 \mathrm{~Kb}$. This finding confirms the existence of a subgroup of cases with a very stable karyotype that are caused by either a unique or very few genetic abnormalities.
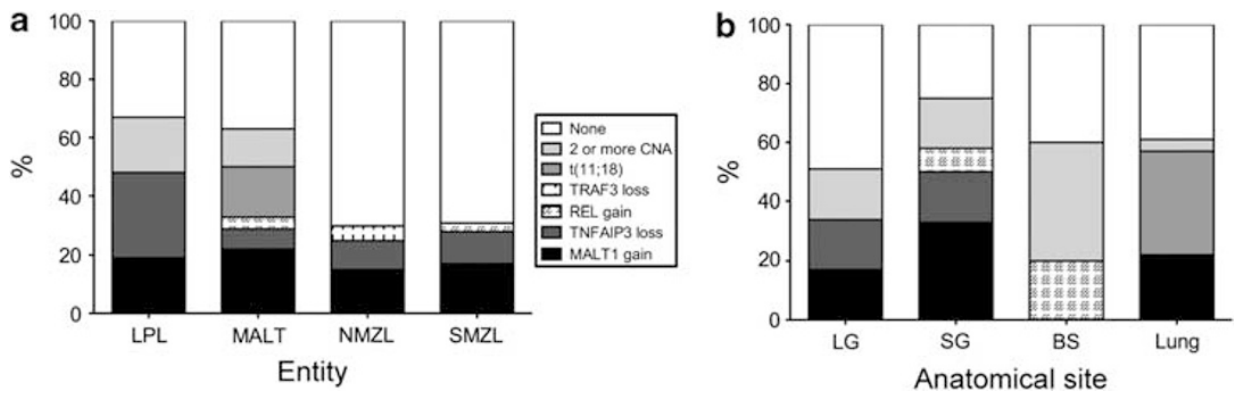

Figure 4 (a) Bar graphic showing the frequency of copy-number abnormality affecting key regulators of the NF- $\mathrm{B}$ signaling pathways per entity. (b) Bar graphic showing the frequency of copy-number abnormality affecting key regulators of the NF-kB signaling pathways in MALT lymphomas depending on the anatomic site of origin. BS, bowel and stomach; LG, lacrimal glands; SG, salivary glands. 
At the level of cell differentiation these tumors are very similar (memory B-cell phenotype, with a tendency to plasmacytic differentiation), but also showing distinct clinical features. A comparative analysis across entities exposes a similar scenario at the genomic level, with the presence of a common group of copy-number abnormalities shared by several, or all of the entities with few disease-specific abnormalities. In fact, the genomic similarities are not restricted to the analyzed lymphoma entities but are also common to a larger group of low-grade B-cell malignancies. Indeed, lymphoplasmacytic and splenic marginal zone lymphomas are affected by the most common copy-number abnormalities observed in chronic lymphocytic leukemia, such as deletions of $6 \mathrm{q}, 11 \mathrm{q} 21-\mathrm{q} 22,13 \mathrm{q} 14.3,17 \mathrm{p} 13$ and gains of chromosome $12 .^{34}$

Even though no distinctive disease-specific copynumber abnormalities have been identified across the analyzed lymphoma entities, the overall genomic profiles may help us to better differentiate between these entities. Although deletion of 6q23q24 and partial gain of chromosomes 3 and 18, observed alone or in combination, are present in almost $50 \%$ of patients across all entities, these abnormalities are more commonly observed in MALT lymphomas and lymphoplasmacytic lymphoma than in splenic or nodal marginal zone lymphoma. Deletion of 11q21-q22 was observed in lymphoplasmacytic lymphoma, splenic and nodal marginal zone lymphoma, but not in MALT lymphomas. Focal deletion of 13q14.3 including MIRN15A and MIRN16-1 was only found in lymphoplasmacytic and splenic marginal zone lymphoma. Deletion of 17p (including TP53) was observed in lymphoplasmacytic lymphoma and splenic, but not in nodal marginal zone or MALT lymphomas. Deletion 7q32.1-q33, previously described in splenic marginal zone lymphoma, was also found at low prevalence in MALT lymphomas and lymphoplasmacytic lymphoma, but not in nodal marginal zone lymphoma. These abnormalities, if validated in future studies, will not only help to elucidate pathogenesis of these lymphoma entities, but may be used for diagnosis confirmation.

We have confirmed previous findings of the importance of the NF- $\mathrm{BB}$ pathway in the pathogenesis of these lymphomas. In MALT lymphomas, at least four translocations lead to the overexpression of MALT1 or BCL10, thus unifying all under a common pathogenesis pathway, which results in the constitutive activation of the NF- $\mathrm{BB} .{ }^{14-16}$ Despite the characterization of several translocations leading to the constitutive activation of the NF- $\mathrm{B}$ signaling pathway, there is also a significant group of MALT lymphoma cases without any identified translocation. Remarkably, $55 \%$ of cases without translocations are also characterized by abnormalities affecting regulators of the NF- $\mathrm{BB}$ pathway, such as 1-3 copy gains of the positive regulators MALT1 (18q21) and REL (2p15-p16) and mono/biallelic deletions of the negative regulator TNFAIP3 (6q23q24). A previous study showed recurrent gains of TRAF2 in MALT, ${ }^{33}$ but we did not find abnormalities affecting this gene in our cohort.

TNFAIP3/A20 gene is a negative regulator of the $\mathrm{NF}-\kappa \mathrm{B}$ pathway by controlling RIP, TRAF6 and $N E M O^{35,36}$ and its inactivation contributes to lymphomagenesis by promoting constitutive activation of the NF- $\mathrm{KB}$ and enhanced cell survival. Consistent with the tumor-suppressive function of TNFAIP3, reintroduction of the wild-type A20 into A20-deficient lymphoma cell lines suppressed cell growth, promoted apoptosis and downregulated NF-kB activity. ${ }^{37-40}$ We and others have recently demonstrated the presence of TNFAIP3 deletions and mutations in a broad set of B-cell diseases. ${ }^{12,21,37-42}$ In this study we confirmed the presence of homozygous deletions in MALT lymphomas, nodal marginal zone and lymphoplasmacytic lymphoma as well as focal monoallelic deletions in all analyzed lymphoma entities. Contrary to other recent studies we did not find mutations in TNFAIP3 leading to the inactivation of the second allele. ${ }^{21,37,38,43}$

Interestingly, the protease MALT1 interacts with A20, by cleaving and inactivating it, thus enhancing NF- $\mathrm{\kappa B}$ signaling. ${ }^{44}$ Thus, alterations affecting these two regulators may act synergistically in the activation of the NF- $\kappa B$ pathway. As the list of cytogenetic alterations involving other $\mathrm{NF}-\kappa \mathrm{B}$ pathway members currently implicated in MALT lymphoma grows, the unifying theory ${ }^{14-16}$ receives strong support and suggests that there is also a synergistic interplay between several abnormalities affecting the NF- $\mathrm{BB}$ pathway other than the translocations found in MALT lymphoma. It might be hypothesized that these additional abnormalities, together with the chronic immunological stimulations, leads to biological consequences similar to those caused by the chromosomal translocation associated with MALT lymphoma. These findings provide a rationale for exploring proteasome inhibitor-based therapeutic strategies for patients with recurrent or refractory disease.

More intriguing is the role the NF- $\mathrm{BB}$ pathway has in lymphoplasmacytic lymphoma, where no translocations have been identified. Besides copy-number abnormalities affecting TNFAIP3, MALT1 and $R E L$, focal deletions and mutations affecting TRAF3 were identified in this and in previous studies involving other B-cell malignancies. ${ }^{21,29,45}$ Remarkably, $67 \%$ of lymphoplasmacytic lymphoma cases show copy-number abnormalities affecting this pathway, thus highlighting the role of the NF-кB pathway in lymphoplasmacytic lymphoma, whether associated with Waldenström's macroglobulinemia or not. This does not seem to be the case in nodal or marginal zone lymphoma, where chromosomal abnormalities affecting the NF- $\mathrm{BB}$ pathway were identified at a significantly lower prevalence, thereby suggesting that the activation of this pathway does not have the central role observed in MALT lymphomas and lymphoplasmacytic lymphoma. 
In conclusion, we performed a comprehensive high-resolution genomic analysis in a large series of marginal zone lymphomas and lymphoplasmacytic lymphomas to better dissect the genetic basis of these entities. Although the entities share a common set of genomic abnormalities they are also characterized by the presence of genes and cellular pathways differentially affected. Elucidation of the genetic alterations contributing to the pathogenesis of the analyzed non-Hodgkin lymphoma subtypes may guide design of specific therapeutic approaches.

\section{Acknowledgement}

This work was supported by a University of Iowa/ Mayo Clinic Lymphoma SPORE grant from the National Cancer Institute/National Institutes of Health (NCI/NIH; CA097274).

\section{Disclosure/conflict of interest}

$\mathrm{RF}$ is a consultant for Genzyme, Medtronic, BMS, Amgen, Otsuka, Celgene, Intellikine and Lilly (all less than $\$ 10000)$. $R F$ receives research support from Cylene, Onyx and Celgene.

Author contributions. EB, AD, RF designed the study; EB, WC, JJK, GH, MB performed the research; $\mathrm{AD}, \mathrm{JM}, \mathrm{DB}$, ISL, TW contributed with patient samples; EB, RF, ISL, JMM wrote the paper; all authors reviewed and gave final approval of the manuscript.

\section{References}

1 Harris NL, Jaffe ES, Diebold J, et al. The World Health Organization classification of neoplastic diseases of the hematopoietic and lymphoid tissues. Report of the Clinical Advisory Committee meeting, Airlie House, Virginia, November, 1997. Ann Oncol 1999;10: 1419-1432.

2 Jaffe ES, Harris NL, Diebold J, et al. World Health Organization classification of neoplastic diseases of the hematopoietic and lymphoid tissues. A progress report. Am J Clin Pathol 1999;111:S8-12.

3 Fonseca R, Hayman S. Waldenstrom macroglobulinaemia. Br J Haematol 2007;138:700-720.

4 Auer IA, Gascoyne RD, Connors JM, et al. t(11;18)(q21;q21) is the most common translocation in MALT lymphomas. Ann Oncol 1997;8:979-985.

5 Ott G, Katzenberger T, Greiner A, et al. The t(11;18)(q21;q21) chromosome translocation is a frequent and specific aberration in low-grade but not high-grade malignant non-Hodgkin's lymphomas of the mucosaassociated lymphoid tissue (MALT-) type. Cancer Res 1997;57:3944-3948.

6 Wotherspoon AC, Finn TM, Isaacson PG. Trisomy 3 in low-grade B-cell lymphomas of mucosa-associated lymphoid tissue. Blood 1995;85:2000-2004.

7 Streubel B, Lamprecht A, Dierlamm J, et al. T(14;18)(q32;q21) involving IGH and MALT1 is a frequent chromosomal aberration in MALT lymphoma. Blood 2003;101:2335-2339.

8 Willis TG, Jadayel DM, Du MQ, et al. Bcl10 is involved in $\mathrm{t}(1 ; 14)(\mathrm{p} 22 ; \mathrm{q} 32)$ of MALT B cell lymphoma and mutated in multiple tumor types. Cell 1999;96:35-45.

9 Wotherspoon AC, Pan LX, Diss TC, et al. Cytogenetic study of B-cell lymphoma of mucosa-associated lymphoid tissue. Cancer Genet Cytogenet 1992;58:35-38.

10 Ferreira BI, Garcia JF, Suela J, et al. Comparative genome profiling across subtypes of low-grade B-cell lymphoma identifies type-specific and common aberrations that target genes with a role in B-cell neoplasia. Haematologica 2008;93:670-679.

11 Rinaldi A, Mian M, Chigrinova E, et al. Genome wide DNA-profiling of marginal zone lymphomas identifies subtype-specific lesions with an impact on the clinical outcome. Blood. 2011;117:1595-1604.

12 Novak U, Rinaldi A, Kwee I, et al. The NF-\{kappa\}B negative regulator TNFAIP3 (A20) is inactivated by somatic mutations and genomic deletions in marginal zone lymphomas. Blood 2009;113:4918-4921.

13 Starostik P, Patzner J, Greiner A, et al. Gastric marginal zone B-cell lymphomas of MALT type develop along 2 distinct pathogenetic pathways. Blood 2002;99:3-9.

14 Farinha P, Gascoyne RD. Molecular pathogenesis of mucosa-associated lymphoid tissue lymphoma. J Clin Oncol 2005;23:6370-6378.

15 Isaacson PG, Du MQ. MALT lymphoma: from morphology to molecules. Nat Rev Cancer 2004;4:644-653.

16 Lucas PC, Yonezumi M, Inohara N, et al. Bcl10 and MALT1, independent targets of chromosomal translocation in malt lymphoma, cooperate in a novel NFkappa B signaling pathway. J Biol Chem 2001;276: 19012-19019.

17 Mateo M, Mollejo M, Villuendas R, et al. 7q31-32 allelic loss is a frequent finding in splenic marginal zone lymphoma. Am J Pathol 1999;154:1583-1589.

18 Sole F, Salido M, Espinet B, et al. Splenic marginal zone B-cell lymphomas: two cytogenetic subtypes, one with gain of $3 q$ and the other with loss of $7 q$. Haematologica 2001;86:71-77.

19 Salido M, Baro C, Oscier D, et al. Cytogenetic aberrations and their prognostic value in a series of 330 splenic marginal zone B-cell lymphomas: a multicenter study of the Splenic B-Cell Lymphoma Group. Blood 2010;116:1479-1488.

20 Baldini L, Fracchiolla NS, Cro LM, et al. Frequent p53 gene involvement in splenic B-cell leukemia/lymphomas of possible marginal zone origin. Blood 1994;84:270-278.

21 Braggio E, Keats JJ, Leleu X, et al. Identification of copy number abnormalities and inactivating mutations in two negative regulators of nuclear factor-kappaB signaling pathways in Waldenstrom's macroglobulinemia. Cancer Res 2009;69:3579-3588.

22 Schop RF, Kuehl WM, Van Wier SA, et al. Waldenstrom macroglobulinemia neoplastic cells lack immunoglobulin heavy chain locus translocations but have frequent $6 q$ deletions. Blood 2002;100:2996-3001.

23 Terre C, Nguyen-Khac F, Barin C, et al. Trisomy 4, a new chromosomal abnormality in Waldenstrom's macroglobulinemia: a study of 39 cases. Leukemia 2006;20:1634-1636.

24 Swerdlow SH, Campo E, Harris NL, et al. WHO Classification of Tumors of Haematopoietic and Lymphoid Tissues 4th Edn 2008;2:439.

25 Owen RG. Developing diagnostic criteria in Waldenstrom's macroglobulinemia. Semin Oncol 2003;30:196-200. 
26 Owen RG, Treon SP, Al-Katib A, et al. Clinicopathological definition of Waldenstrom's macroglobulinemia: consensus panel recommendations from the Second International Workshop on Waldenstrom's Macroglobulinemia. Semin Oncol 2003;30: $110-115$.

27 Chng WJ, Remstein ED, Fonseca R, et al. Gene expression profiling of pulmonary mucosa-associated lymphoid tissue lymphoma identifies new biologic insights with potential diagnostic and therapeutic applications. Blood 2009;113:635-645.

28 Lipson D, Aumann Y, Ben-Dor A, et al. Efficient calculation of interval scores for DNA copy number data analysis. J Comput Biol 2006;13:215-228.

29 Keats JJ, Fonseca R, Chesi M, et al. Promiscuous mutations activate the noncanonical NF-kappaB pathway in multiple myeloma. Cancer Cell 2007;12:131-144.

30 Largo C, Saez B, Alvarez S, et al. Multiple myeloma primary cells show a highly rearranged unbalanced genome with amplifications and homozygous deletions irrespective of the presence of immunoglobulinrelated chromosome translocations. Haematologica 2007;92:795-802.

31 Hartmann EM, Campo E, Wright G, et al. Pathway discovery in mantle cell lymphoma by integrated analysis of high-resolution gene expression and copy number profiling. Blood 2010;116:953-961.

32 Lenz G, Wright GW, Emre NC, et al. Molecular subtypes of diffuse large B-cell lymphoma arise by distinct genetic pathways. Proc Natl Acad Sci USA 2008;105:13520-13525.

33 Zhou Y, Ye H, Martin-Subero JI, et al. Distinct comparative genomic hybridisation profiles in gastric mucosa-associated lymphoid tissue lymphomas with and without $\mathrm{t}(11 ; 18)(\mathrm{q} 21 ; \mathrm{q} 21)$. Br J Haematol 2006; 133:35-42.

34 Kay NE, Eckel-Passow JE, Braggio E, et al. Progressive but previously untreated CLL patients with greater array CGH complexity exhibit a less durable response to chemoimmunotherapy. Cancer Genet Cytogenet 2010;203:161-168.
35 Boone DL, Turer EE, Lee EG, et al. The ubiquitinmodifying enzyme A20 is required for termination of Toll-like receptor responses. Nat Immunol 2004;5: 1052-1060.

36 Wang YY, Li L, Han KJ, et al. A20 is a potent inhibitor of TLR3- and Sendai virus-induced activation of NFkappaB and ISRE and IFN-beta promoter. FEBS Lett 2004;576:86-90.

37 Compagno M, Lim WK, Grunn A, et al. Mutations of multiple genes cause deregulation of NF-kappaB in diffuse large B-cell lymphoma. Nature 2009;459:717-721.

38 Honma K, Tsuzuki S, Nakagawa M, et al. TNFAIP3/ A20 functions as a novel tumor suppressor gene in several subtypes of non-Hodgkin lymphomas. Blood 2009.

39 Kato M, Sanada M, Kato I, et al. Frequent inactivation of A20 in B-cell lymphomas. Nature 2009;459:712-716.

40 Schmitz R, Hansmann ML, Bohle V, et al. TNFAIP3 (A20) is a tumor suppressor gene in Hodgkin lymphoma and primary mediastinal B cell lymphoma. J Exp Med 2009;206:981-989.

41 Chanudet E, Ye H, Ferry J, et al. A20 deletion is associated with copy number gain at the TNFA/B/C locus and occurs preferentially in translocation-negative MALT lymphoma of the ocular adnexa and salivary glands. J Pathol 2009;217:420-430.

42 Honma K, Tsuzuki S, Nakagawa M, et al. TNFAIP3 is the target gene of chromosome band 6q23.3-q24.1 loss in ocular adnexal marginal zone B cell lymphoma. Genes Chromosomes Cancer 2008;47:1-7.

43 Braggio E, Keats JJ, Leleu X, et al. High-resolution genomic analysis in Waldenstrom's macroglobulinemia identifies disease-specific and common abnormalities with marginal zone lymphomas. Clin Lymphoma Myeloma 2009;9:39-42.

44 Thome M. Multifunctional roles for MALT1 in T-cell activation. Nat Rev Immunol 2008;8:495-500.

45 Nagel I, Bug S, Tonnies H, et al. Biallelic inactivation of TRAF3 in a subset of B-cell lymphomas with interstitial del(14)(q24.1q32.33). Leukemia 2009;23: 2153-2155.

Supplementary Information accompanies the paper on Modern Pathology website (http://www.nature.com/ modpathol) 